\title{
PRÁCTICAS Y CONCEPTO DE EVALUACIÓN PREVALENTES EN DOCENTES DE LOS NIVELES PRIMARIO Y SECUNDARIO
}

\section{PREVALENT PRACTICES AND CONCEPT OF STUDENT ASSESSMENT IN TEACHERS OF THE PRIMARY AND SECONDARY LEVELS}

\author{
Sonia Patricia Krumm \\ Universidad Adventista del Plata \\ soniakrumm@gmail.com \\ https://orcid.org/0000-0001-9499-0524 \\ Doris Cairus \\ Universidad Adventista del Plata \\ doriscc@gmail.com \\ https://orcid.org/0000-0001-8075-7174 \\ Josué Quinde \\ Universidad Adventista del Plata \\ josuequinde@yahoo.com \\ https://orcid.org/0000-0002-2928-8787
}

\begin{abstract}
RESUMEN
El propósito de este estudio fue describir las prácticas de evaluación más utilizadas y el concepto de evaluación predominante en 522 docentes de los niveles primario y secundario en instituciones argentinas de educación privada. Se les administró un cuestionario con preguntas (a) sociodemográficas, (b) de frecuencia de utilización de las prácticas de evaluación $y$ (c) abiertas, que exploran el concepto de evaluación de los docentes. Se realizaron análisis descriptivos para hallar las prácticas de evaluación más utilizadas, además de un análisis cualitativo para descubrir los conceptos de evaluación predominantes. Se encontró que las formas más frecuentes de evaluar son los siguientes: (a) en el primer ciclo de primaria, exámenes escritos con ejercicios similares a los dados en clase, trabajos prácticos individuales y exámenes orales; (b) en el segundo ciclo de primaria, trabajos prácticos individuales, exámenes escritos con ejercicios similares a los dados en clase y exámenes orales; (c) en el nivel secundario, trabajos prácticos individuales, trabajos prácticos grupales y exámenes escritos con ejercicios similares a los dados en clase. El instrumento menos utilizado, en los tres casos, es el portafolio. En cuanto al concepto de evaluación, se encontraron 25 categorías. Las 10 más frecuentes destacan la evaluación como medio para visibilizar lo invisible y observar detalles; verificación de contenidos conceptuales; detección de errores, nivelación y
\end{abstract}


corrección; mejora, cambio y oportunidad; seguimiento de un proceso; medición, manera de conocer a un estudiante en profundidad; construcción; sinónimo de autoevaluación; aprendizaje. Las prácticas de evaluación que predominan en los tres niveles pueden ser catalogadas como tradicionales e incompletas para la evaluación de competencias. Los conceptos de evaluación que predominan revelan una tensión entre la evaluación auténtica y la evaluación tradicional o de medición.

Palabras clave: concepto de evaluación, instrumentos de evaluación, prácticas de evaluación, niveles de educación primaria y secundaria

\section{ABSTRACT}

The purpose of this study was to describe the most used assessment practices and the predominant assessment concept in 522 primary and secondary school teachers in Argentine private educational institutions. A questionnaire with sociodemographic questions, information about the frequency of use of assessment practices, and open-ended questions which explore teachers' concept of assessment was administered. Descriptive analyzes were performed to find the most widely used evaluation practices, in addition to qualitative analysis, to discover the predominant evaluation concepts. It was found that the most common forms of evaluation are the following: (a) in the first cycle of primary school, written exams with exercises similar to those given in class, individual practical work, and oral exams; (b) in the second cycle of primary school, individual practical assignments, written exams with exercises similar to those given in class, and oral exams; (c) at the secondary level, individual practical assignments, group practical assignments, and written exams with exercises similar to those given in class. The least used instrument is the portfolio. Regarding the concept of evaluation, 25 categories were found. The 10 most frequent categories highlight evaluation as a means of making the invisible visible and observing details; verification of conceptual content; error detection, leveling and correction; improvement, change and opportunity; monitoring of a process; measurement, the way of knowing a student in-depth; building; synonymous with self-evaluation; learning. The evaluation practices that predominate in the three levels can be classified as traditional and incomplete for the evaluation of competences. The prevailing assessment concepts reveal a tension between authentic assessment and traditional or measurement assessment.

Keywords: concept of assessment, assessment instruments, assessment practices, primary and secondary education levels

\section{Introducción}

La evaluación suele ser concebida como un proceso trabajoso, que no siempre deviene en satisfacción profesional; sin embargo, no se puede entender el proceso de enseñanza-aprendizaje sin una evaluación formativa y continua que permita el desarrollo y mejora del estudiante y del docente. Gran parte de las frustraciones en el terreno de la evaluación están relacionadas con errores en el proceso de enseñanza y un mal manejo de los procesos de evaluación: conceptualizaciones erróneas de lo que implica 
evaluar, elección incorrecta de los instrumentos de evaluación, su aplicación parcial o errónea y desaprovechamiento de los resultados para producir mejoras.

Luego de una revisión de diversos autores, Cabezas Gómez, González Barberá y Carpintero Molina (2009) concluyeron que la evaluación es un aspecto fundamental y tremendamente complejo que condiciona la educación y que, por esa complejidad, requiere una profunda reflexión en torno a parámetros de carácter teórico, técnico-práctico y ético.

Santos Guerra (2007) afirma que la evaluación es un proceso ético y político que suele disfrazarse como un proceso técnico y científico. Pero esta pretendida asepsia puede esconder riesgos que conviene descubrir para no caer en un autoengaño. Por eso es necesario desentrañar qué se entiende por evaluación y qué acciones y habilidades son requeridos para que sea efectiva.

Por otra parte, es necesario comprender, a los fines de esta investigación, cómo se integran en un mismo proceso la enseñanza, el aprendizaje y la evaluación. La enseñanza para la comprensión (Perkins, 2010) proviene de un enfoque constructivista que incentiva la capacidad de pensar y actuar en forma flexible, y una interiorización profunda de lo que esos conocimientos implican, lo cual permite al que aprende la transformación y aplicación de dichos conocimientos a su realidad. Este proceso de transformación en la enseñanza se encuentra estrechamente vinculado con la evaluación y las acciones que de ella se desprenden. No se puede hablar de un cambio en la enseñanza sin involucrar la evaluación como parte esencial del proceso. Pero la evaluación, como tarea inherente del que educa, no solo es poco apreciada, sino que constituye una de las competencias más difíciles de lograr en forma efectiva. Zabalza (2014) afirma que es casi natural que los estudiantes odien la evaluación, pero que los profesores no deberían detestarla. Por ser una competencia básica de la profesión docente, debería ser una de las competencias que los estudiantes -futuros profesores- desarrollen en mayor profundidad durante su formación y que los docentes consolidados continúen desarrollando a lo largo de sus carreras.

Quizá esta antipatía generalizada por ser evaluados y ser evaluadores tenga su explicación en la incomodidad que produce la exposición a la observación o el poner en tela de juicio las acciones de otros. La valoración, juicio, observación y comparación con estándares siempre producirá incomodidad, sostiene Rafaghelli (2009), incomodidad que solo se mitiga -aunque nunca desaparece totalmente- cuando se aclaran los referentes. Una propuesta para aclarar estos referentes es, en primer lugar, reconocer la complejidad de las prácticas evaluativas y desnaturalizar los referentes: reflexionar acerca de las creencias que rodean a los actos de enseñar, aprender y evaluar. Es en esta reflexión profunda de lo que se cree acerca del ser humano, del valor de un estudiante, del rol del enseñante, de los poderes, deberes y derechos que tiene cada una de las partes, donde se puede comenzar a esbozar ciertas convicciones acerca del complejo acto de evaluar.

Sobre estas construcciones que son muy personales, pero que deberán ser compartidas, discutidas y consensuadas al interior de una institución, es que se erigirán principios o lineamientos generales para buscar una política de evaluación coherente y estable (pero flexible) a fin de lograr una gestión efectiva en el ramo. 


\section{Representaciones mentales}

Parte de las variables observadas en esta investigación han sido las representaciones mentales. Las construcciones conceptuales son importantes porque las creencias y convicciones modelan las acciones. Estas construcciones personales se llaman "representaciones mentales" y, cuando son construcciones compartidas, "representaciones sociales".

La representación mental es una "forma material o simbólica de dar cuenta de algo real en su ausencia" (Arbeláez Gómez, 2001, p. 87). Implica una construcción realizada por el sujeto, en intercambio con un contexto social. Las representaciones "se organizan en estructuras conceptuales, procedimentales y actitudinales para darle sentido a la interioridad y exterioridad de su entorno, con miras al dominio, la intervención, el control y la transformación del mismo".

En el caso de los docentes, estas estructuras constituyen el sustrato cognitivo que funciona como referente para la toma de decisiones que inciden en la calidad del aprendizaje de los alumnos (Prieto y Contreras, 2008). De allí la importancia de realizar un esfuerzo por comprender de dónde provienen las conceptualizaciones que sostienen las acciones de los docentes, pues, si se logra identificar la representación mental, existe mayor posibilidad de que se logre entender y resignificar la acción.

Con respecto a la evaluación, se ha encontrado que los profesores que adoptan una perspectiva tradicional, concibiéndola como un instrumento de control que brinda información con un alto grado de objetividad, tienden a enfatizar las respuestas correctas y las tareas sencillas (Stiggins, 2004, citado en Prieto y Contreras, 2008). Quienes conciben la evaluación como un aporte introspectivo sobre la propia docencia que les permite reorientar su tarea, relevando aspectos formativos, tienden a enfatizar la comprensión por sobre la reproducción, incentivando la construcción del conocimiento con los alumnos y brindándoles mayor participación (Durán, 2001, citado en Prieto y Contreras, 2008).

Las representaciones exhiben cierta estabilidad y pueden incluso permanecer ocultas para el propio sujeto. "Los sujetos que no conocen sus propias representaciones actúan guiados por unas prácticas socializadas, que funcionan en últimas, como estereotipos... quedando ocultas a su razón y a su crítica las verdaderas justificaciones ideológicas de sus actuaciones" (Arbeláez Gómez, 2001, p. 93). Se desprende de esto que, a menos que se logre explicitar la representación, no se podrá modificar, ya que no se puede operar sobre lo desconocido. La implicación para la docencia es clara; cualquier intento de mejora del proceso de evaluación que no procure que los docentes tomen conciencia sobre los aspectos implícitos que constituyen el fundamento de sus decisiones, tendrá poco efecto.

En cuanto a las representaciones sociales, el conocimiento aquí no surge de la racionalidad individual, sino a partir de una construcción social que se incorpora en cada persona y orienta comportamientos, prácticas sociales y decisiones que tienen lugar en las experiencias cotidianas. Esto muestra que el saber, más que intelectual, es de carácter simbólico y emocional, y está relacionado con las vivencias de cada contexto social, por lo cual quedan teñidas de la subjetividad personal. De esta manera, las representaciones sociales también pueden llegar a ser representaciones mentales individuales (Moreno Acero, 
Lozano Ramírez, Pineda Ramos y Ortiz Quiroz, 2016).

La percepción de lo que es bueno y lo que es malo, lo que es correcto e incorrecto puede ser subjetiva y frágil. Esto tiñe cualquier acción humana de subjetividad y es difícil llegar a acuerdos. En este sentido es que la evaluación es un campo complejo de abordar, donde se dificulta el logro de consensos (Rafaghelli, 2009).

¿Por qué las representaciones mentales y sociales son tan diversas y subjetivas? Porque están basadas en creencias, las cuales pueden definirse como "el repertorio de supuestos y respuestas rutinarias a los problemas profesionales que, generalmente no tienen base teórica que las sustenten, pero configuran el comportamiento de los profesores" (Anijovich y Capelletti, 2017, p. 41).

Finalmente, todas estas creencias, supuestos y representaciones mentales acerca de la evaluación pueden inscribirse en un enfoque conductista o constructivista: una evaluación que valora todos los aspectos del proceso o que es más aséptica y rigurosa, centrada en los resultados. Anijovich y Capelletti (2017) citan investigaciones como las de Señoriño et al. (2012) y Mellado Hernández y Chaucono Catrinao (2015), quienes ilustran cómo los docentes, e incluso los estudiantes, se acercan más a una concepción o la otra, dependiendo de sus creencias y experiencias personales y sociales. Anijovich y Capelletti (2017) piensan que las creencias acerca de la evaluación pueden agruparse en dos enfoques: el conductista y el constructivista.

El enfoque conductista es entendido como medición de resultados de aprendizaje al final de un período de ejecución de la enseñanza para verificar el dominio de contenidos y calificar con el propósito de acreditación. El único agente evaluador, el profesorado, otorga escasa retroalimentación y concibe el error con una connotación negativa. (p. 49)

El enfoque constructivista considera a la evaluación como "una instancia para aprender, por tanto, tiene relevancia la función formativa y el error se considera una fuente de aprendizaje. Facilita la autorregulación del aprendizaje a través de la retroalimentación oportuna y constante del estudiante" (p. 49).

\section{Las prácticas de evaluación auténtica}

Las representaciones mentales de los conceptos se materializan en las acciones o prácticas que los docentes realizan para lograr los objetivos educacionales. Es por esto que la forma de entender la evaluación condiciona el proceso de enseñanza y aprendizaje. Este proceso es complejo, extendido y está atravesado por múltiples factores personales, sociales, psicológicos y políticos. Santos Guerra (2017) piensa que la dimensión sociológica de la evaluación la convierte en una criba que selecciona personas al permitir o impedir su avance dentro de las siguientes etapas del proceso. Por otra parte, las repercusiones psicológicas que tiene la evaluación influyen en la configuración del autoconcepto de los estudiantes. Además, en su naturaleza política y ética, la evaluación se modifica como constructo social y cumple con funciones que les interesan a algunos y perjudican a otros.

Estas razones y muchas otras ameritan un estudio de una evaluación auténtica, genuina o legítima. En las discusiones acerca de qué funciona mejor 


\section{PRÁCTICAS Y CONCEPTO DE EVALUACIÓN PREVALENTES}

para que la evaluación cumpla con su propósito, se ha encontrado que una evaluación auténtica "proporciona instrumentos que van más allá del análisis del conocimiento declarativo, esto es, los aspectos conceptuales que los alumnos enuncian. Incluye también la observación y valoración de lo que los alumnos demuestran que saben hacer, pensar y resolver" (Anijovich y González, 2011, p. 13). La evaluación auténtica lleva a la comprensión, tanto de quien aprende como del que enseña. "Permite contemplar la heterogeneidad de los estudiantes y la posibilidad de que todos logren aprender en tanto se les ofrezcan actividades variadas en las que sea posible optar y tomar decisiones para resolver problemas cotidianos" (p. 15).

La evaluación auténtica

intenta averiguar qué sabe el estudiante o qué es capaz de hacer, utilizando diferentes estrategias y procedimientos evaluativos. Se fundamenta en el hecho que existe un espectro mucho más amplio de desempeños que el estudiante puede mostrar a diferencia del conocimiento limitado que se puede evidenciar mediante un examen oral o escrito ya sea de respuesta breve o extensa. (Ahumada, 2005, p. 12)

Según Vallejo Ruiz y Molina Saorín (2014), para que la evaluación sea auténtica, debe explorar los aprendizajes que requieren habilidades cognitivas y acciones complejas, desarrollar tareas que representen tanto el contenido como las habilidades y de esta manera conjuguen la enseñanza con la evaluación, proporcionar a los alumnos el apoyo para que comprendan las expectativas que se tienen de aprendizaje y logren realizarlas, comunicar con claridad los criterios consensuados según los cuales se juzgarán los logros e incluir espacios de reflexión sobre los aprendizajes logrados, el camino recorrido y los instrumentos utilizados para realimentar y mejorar la práctica.

Para que la evaluación sea auténtica, deben existir acuerdos institucionales y consensos que permitan aplicar criterios transversales. Santos Guerra (2007) explica una serie de principios básicos que permiten una gestión de la evaluación eficiente. En una educación democrática y compartida, se podrá estar de acuerdo o no con ellos, o ubicarlos en diversas jerarquías; aun así, son útiles para interpelarnos e intentar construir, sobre las subjetividades naturales de cualquier institución, un conjunto de principios que ordenen la reflexión y la praxis del acto de evaluar.

1. La evaluación es un fenómeno moral, no meramente técnico.

2. La evaluación ha de ser un proceso y no un acto aislado.

3. Es preciso que la evaluación sea un proceso participativo.

4. La evaluación tiene un componente corroborador y otro atributivo.

5. El lenguaje sobre la evaluación nos sirve para entendernos y también para confundirnos.

6. Para que la evaluación tenga rigor ha de utilizar instrumentos diversos.

7. La evaluación es un catalizador de todo proceso de enseñanza y aprendizaje.

8. El contenido de la evaluación ha de ser complejo y globalizador.

9. Para evaluar hace falta tener un conocimiento especializado del proceso de enseñanza-aprendizaje.

10. La evaluación tiene que servir para el aprendizaje.

11. Es importante hacer metaevaluación, o lo que es lo mismo, evaluar las evaluaciones. 
12. La evaluación no debe ser un acto individualista sino colegiado.

En el marco de evaluación propuesto por Álvarez Méndez (2000), existen cuatro principios para la funcionalidad de la evaluación como aprendizaje, las cuales abarcan casi en su totalidad a los principios que propone Santos Guerra (2007):

1. Estrechar la relación entre enseñanza y evaluación: "Dime cómo enseñas y te diré cómo has de evaluar".

2. La introducción de la perspectiva cualitativa en la evaluación significa un cambio estructural radical que obliga a un cambio en las estructuras escolares que lleva a un replanteamiento de los roles asignados a los sujetos comprometidos en la educación.

3. Desde la óptica cualitativa se interpreta la evaluación como una cuestión ética y no solo como cuestión técnica.

4. La evaluación no es un apéndice de la enseñanza, sino parte integral del proceso de enseñanza y aprendizaje, desempeñando funciones de diagnóstico, corrección, comprobación y prevención de necesidades.

En cuanto a las funciones de la evaluación, Elola, Zanelli, Oliva y Toranzos (2010), las sintetizan de esta manera:

1. Función simbólica: se le atribuye a la evaluación, en general, el carácter de fin de etapa o ciclo. Es común que se relacione a la evaluación con un "broche final".

2. Función política: remite a la posibilidad de generar información que se constituye en el fundamento para la toma de decisiones.

3. Función de conocimiento: permite ampliar la comprensión de los procesos complejos en forma sistemática, lo cual incrementa la comprensión del conocimiento.
4. Función de mejoramiento: es una herramienta para mejorar los procesos-objeto de la evaluación. Remite a la efectividad, pertinencia y viabilidad de las propuestas.

5. Función contractual: la evaluación explicita el contrato pedagógico que subyace a la actividad del aula; qué se evalúa, cuándo, cómo y sobre qué criterios. Finalmente establece lo que el docente realmente espera del alumno y lo que este está dispuesto a invertir en esa relación.

6. Función de desarrollo de capacidades: la evaluación promueve el desarrollo de competencias muy valiosas, tanto individuales como colectivas (institucionales).

La comprensión de lo que implica una evaluación auténtica lleva a alinearse a un estilo constructivista, antes que a uno tradicional o conductista. La conciencia acerca de las funciones de la evaluación, la reflexión acerca de los principios y los referentes sobre los cuales se construye el acto de evaluar se constituyen en el punto de partida para repensar las prácticas y realizar propuestas nuevas, que retomen las prácticas constructivas y colaboren con el desprendimiento de acciones de evaluación viciadas que no acompañan el crecimiento personal de los estudiantes y profesionales ni el desarrollo institucional. En síntesis, y en palabras de Anijovich y Cappelletti (2017), si la evaluación no está al servicio de la mejora, no sirve. No hay por qué destinar tiempo ni recursos institucionales si no produce cambios que impulsen el desarrollo del estudiante y del docente.

\section{Método}

La investigación siguió un diseño metodológico mixto, que incluyó los enfoques cuantitativo y cualitativo. 


\section{Objetivos}

El propósito de este estudio fue describir los instrumentos de evaluación más utilizados y el concepto de evaluación que predomina en los docentes de los niveles primario y secundario de un grupo de escuelas privadas en Argentina.

\section{Muestra}

Durante el mes de agosto de 2017 se encuestaron a 522 docentes de instituciones de nivel primario y secundario. El $76 \%$ de la muestra corresponde al género femenino y el $24 \%$ restante, al género masculino. El $46 \%$ se desempeña en el nivel secundario y el $54 \%$ en el nivel primario (primer ciclo $30 \%$ y segundo ciclo 24\%).

\section{Instrumento}

Se utilizó el Cuestionario de Evaluación Integral de Cabezas et al. (2009), al cual se adicionaron preguntas sociodemográficas (género, en qué nivel enseña mayor cantidad de horas, en qué área se desempeña, años de experiencia en la docencia, ubicación geográfica de la institución), de frecuencia de utilización de instrumentos de evaluación y dos preguntas abiertas que exploran el concepto de evaluación de los docentes. Para el presente informe de investigación, sólo se utilizaron los datos sociodemográfi$\cos$, de frecuencia de utilización de instrumentos de evaluación y las dos preguntas abiertas.

Las preguntas abiertas tenían el propósito de explorar las representaciones mentales del concepto de evaluación a través de las siguientes consignas:

1. Si tuvieras que elegir algún objeto de uso cotidiano para representar tu concepto de evaluación, ¿cuál sería? Te ofrecemos algunos a modo de ejemplo (puedes escoger otros que no están en este listado): lupa, colador, nivel de albañil, radiografía, pincel, llave, tijera... otros.

2. Explica por qué ese objeto es el que mejor representa tu concepto de evaluación.

\section{Análisis de datos}

Se utilizó el programa Statistical Package for Social Sciences (SPSS) para la realización de los análisis estadísticos. Se realizaron análisis descriptivos para hallar los instrumentos de evaluación más frecuentemente utilizados. Para estudiar las diferencias en la utilización de las prácticas de evaluación según años de experiencia y nivel educativo, se utilizó el análisis de $\chi^{2}$.

Para analizar las preguntas abiertas, en primer término se realizó un análisis de contenido que permitió encontrar categorías emergentes a partir de las respuestas de los docentes. En una segunda lectura, se reagruparon categorías que apuntaban a funciones similares. Finalmente se describió la frecuencia de aparición de las categorías que están enfocadas en una evaluación de tipo conductista/tradicional o constructivista, obteniendo un panorama general del paradigma en que los docentes operan la evaluación.

\section{Resultados \\ Utilización de prácticas de evaluación}

En el cuestionario se incluyó una lista de 22 prácticas de evaluación de las cuales los profesores podían seleccionar y marcar todas las usadas. Al analizar estos resultados se eligieron las cinco prácticas más utilizadas por cada docente. Se encontró que las formas más frecuentes de evaluar, según el nivel educativo, son las siguientes: 
1. En el primer ciclo de educación primaria, los docentes utilizan más frecuentemente las siguientes prácticas: exámenes escritos con ejercicios para resolver, similares a los dados en clase $(75,3 \%)$, trabajos prácticos individuales $(71,4 \%)$, exámenes orales $(70,8 \%)$, resolución de problemas $(66,9 \%)$, exposiciones orales de un tema investigado (65,6\%).

2. En el segundo ciclo de educación primaria, los docentes utilizan más frecuentemente las siguientes prácticas: trabajos prácticos individuales (89\%), exámenes escritos con ejercicios para resolver, similares a los dados en clase $(85 \%)$, exámenes orales $(80,3 \%)$, exposiciones orales de un tema investigado $(78 \%)$, resolución de problemas $(71,7 \%)$.

3. En el nivel secundario, los docentes utilizan más frecuentemente las siguientes prácticas: trabajos prácticos individuales $(89,6 \%)$, trabajos prácticos grupales $(86,3 \%)$, exámenes escritos con ejercicios para resolver, similares a los dados en clase (80,1\%), exposiciones orales de un tema investigado $(72,6 \%)$, exámenes orales $(68,5 \%)$.

\section{Prácticas de evaluación y niveles educativos}

Hasta aquí se pudieron ver los porcentajes de prácticas de evaluación más frecuentes de cada nivel. A continuación se analizan las prácticas de evaluación más comunes comparando los tres niveles entre sí. Teniendo en cuenta el tipo de prácticas, la Tabla 1 presenta el porcentaje de docentes que las eligieron y el nivel al que pertenecen. De esta manera se puede visualizar en qué nivel es más frecuentemente utilizada cada práctica.

Utilizando el estadístico $\chi^{2}$, se halló que existe asociación estadísticamen- te significativa $(p<, 05)$ entre el nivel educativo y la utilización de las siguientes prácticas de evaluación: dictados, maquetas, elaboración de un producto, cuestionarios, exámenes con preguntas de desarrollo, exámenes de preguntas objetivas, exámenes a libro abierto, coevaluación, resolución de problemas, diagramas/esquemas/mapas conceptuales, resumen o síntesis de contenidos, trabajos prácticos individuales, trabajos prácticos grupales, rúbrica de realimentación.

El análisis de $\chi^{2}$ mostró que en el primer ciclo de primaria se utilizan más que en los otros niveles, los dictados y maquetas como prácticas de evaluación. Las restantes prácticas se utilizan en forma similar a los otros niveles educativos.

Asimismo, el análisis de $\chi^{2}$ indicó que en el nivel secundario se utilizan más que en los otros niveles, las siguientes prácticas de evaluación: elaboración de un producto, cuestionarios, exámenes con preguntas de desarrollo, exámenes con preguntas objetivas, exámenes a libro abierto, coevaluación, resolución de problemas, diagramas/esquemas/mapas conceptuales, resumen o síntesis de contenidos, trabajos prácticos individuales, trabajos prácticos grupales y rúbricas de realimentación.

\section{Prácticas de evaluación y años de experiencia docente}

Se categorizó la experiencia docente en cuatro grupos: menos de 5 años, de 5 a 10 años, de 10 a 15 años, y más de 15 años para buscar la relación con los instrumentos más utilizados. La mayoría de la muestra (58\%) no tiene más de 10 años de experiencia. La Tabla 2 muestra la distribución de la muestra según los años de experiencia. 


\section{PRÁCTICAS Y CONCEPTO DE EVALUACIÓN PREVALENTES}

Tabla 1

Prácticas de evaluación según el nivel educativo

\begin{tabular}{|c|c|c|c|c|}
\hline Práctica & $\begin{array}{c}\text { Primario } \\
\text { Primer ciclo }\end{array}$ & $\begin{array}{c}\text { Primario } \\
\text { Segundo ciclo }\end{array}$ & Secundario & $\begin{array}{c}\text { Comparación } \\
p\end{array}$ \\
\hline $\begin{array}{l}\text { 1. Examen escrito con ejercicios simila- } \\
\text { res a los dados en la clase }\end{array}$ & $27,82 \%$ & $25,90 \%$ & $46,28 \%$ & , 129 \\
\hline 2. Dictados & $41,10 \%$ & $27,54 \%$ & $31,36 \%$ &, 000 \\
\hline 3. Maquetas & $39,58 \%$ & $38,02 \%$ & $22,40 \%$ &, 000 \\
\hline 4. Elaboración de un producto & $38,95 \%$ & $21,05 \%$ & $40,00 \%$ &, 002 \\
\hline 5. Cuestionarios & $22,11 \%$ & $26,07 \%$ & $51,82 \%$ &, 000 \\
\hline 6. Examen oral & $28,99 \%$ & $27,13 \%$ & $43,88 \%$ &, 051 \\
\hline $\begin{array}{l}\text { 7. Exposición oral de un tema investi- } \\
\text { gado }\end{array}$ & $26,93 \%$ & $26,40 \%$ & $46,67 \%$ &, 067 \\
\hline $\begin{array}{l}\text { 8. Exámenes con preguntas de respuesta } \\
\text { breve }\end{array}$ & $26,02 \%$ & $25,08 \%$ & $48,90 \%$ &, 087 \\
\hline 9. Exámenes con preguntas de desarrollo & $15,48 \%$ & $23,01 \%$ & $61,51 \%$ &, 000 \\
\hline $\begin{array}{l}\text { 10. Exámenes de preguntas "objetivas" } \\
\text { (opción múltiple, F/V, corresp., etc.) }\end{array}$ & $22,83 \%$ & $27,95 \%$ & $49,21 \%$ &, 004 \\
\hline 11. Examen a libro abierto & $12,32 \%$ & $29,38 \%$ & $58,29 \%$ &, 000 \\
\hline 12. Autoevaluación & $24,39 \%$ & $28,78 \%$ & $46,83 \%$ &, 056 \\
\hline $\begin{array}{l}\text { 13. Coevaluación (evaluación entre } \\
\text { compañeros) }\end{array}$ & $20,00 \%$ & $24,67 \%$ & $55,33 \%$ & ,006 \\
\hline 14. Resolución de problemas & $31,21 \%$ & $27,58 \%$ & $41,21 \%$ & ,008 \\
\hline $\begin{array}{l}\text { 15. Diagramas, esquemas, mapas con- } \\
\text { ceptuales }\end{array}$ & $12,79 \%$ & $25,58 \%$ & $61,63 \%$ &, 000 \\
\hline 16. Portafolio & $29,03 \%$ & $19,35 \%$ & $51,61 \%$ &, 760 \\
\hline 17. Resumen o síntesis de contenidos & $13,20 \%$ & $28,43 \%$ & $58,38 \%$ &, 000 \\
\hline 18. Trabajos prácticos individuales & $25,06 \%$ & $25,74 \%$ & $49,20 \%$ &, 000 \\
\hline 19. Trabajos prácticos grupales & $24,49 \%$ & $22,98 \%$ & $52,53 \%$ &, 000 \\
\hline 20. Rúbrica de realimentación & $13,64 \%$ & $13,64 \%$ & $72,73 \%$ & ,038 \\
\hline $\begin{array}{l}\text { 21. Lista de cotejo para observación de } \\
\text { desempeño }\end{array}$ & $25,45 \%$ & $20,00 \%$ & $54,55 \%$ & 417 \\
\hline Otro & $22,97 \%$ & $22,97 \%$ & $54,05 \%$ & 290 \\
\hline
\end{tabular}

Tabla 2

Porcentajes de la muestra según categoría de años de experiencia docente

\begin{tabular}{lc}
\hline $\begin{array}{c}\text { Años de experiencia } \\
\text { docente }\end{array}$ & $\begin{array}{c}\text { Porcentaje de la } \\
\text { muestra }\end{array}$ \\
\hline Menos de 5 años & $35 \%$ \\
$5-10$ años & $23 \%$ \\
$10-15$ años & $16 \%$ \\
Más de 15 años & $26 \%$ \\
\hline
\end{tabular}

El estadístico $\chi^{2}$ indicó que existe asociación estadísticamente significativa $(p<, 05)$ entre los años de experiencia y la utilización de las siguientes prácticas de evaluación: cuestionarios, examen a libro abierto, autoevaluación, coevaluación, trabajos prácticos individuales, rúbrica de realimentación. Los docentes que tienen más de 15 años de experiencia tienden a utilizar más los cuestionarios, los trabajos prácticos individuales y las rúbricas que los de los otros rangos de años de experiencia. Los que tienen menos de cinco años de experiencia tienden a 
utilizar menos el examen a libro abierto, la autoevaluación y la coevaluación que los demás rangos de experiencia. La Tabla 3 muestra en detalle estas relaciones.

\section{Representaciones mentales de evaluación}

$\mathrm{Al}$ analizar las respuestas a las preguntas abiertas se tuvieron en cuenta los objetos elegidos para representar el concepto de evaluación junto con su respectiva explicación. Esta asociación es importante, ya que en algunos casos un mismo objeto era utilizado por distintos docentes como una metáfora de una representación conductista/ tradicional de la evaluación o como una representación constructivista de la evaluación. Por ejemplo, el objeto "colador" fue utilizado para explicar a la evaluación como una manera de decantar o delatar lo que los alumnos no

Tabla 3

Prácticas de evaluación y años de experiencia en la docencia

\begin{tabular}{|c|c|c|c|c|c|}
\hline Instrumento & $<5$ años & 5-10 años & 10-15 años & $>15$ años & $\begin{array}{l}\text { Compara- } \\
\text { ción } p\end{array}$ \\
\hline $\begin{array}{l}\text { 1. Examen escrito con ejercicios } \\
\text { similares a los de las clases }\end{array}$ & $33,6 \%$ & $25,2 \%$ & $15,6 \%$ & $25,7 \%$ &, 130 \\
\hline 2. Dictados & $35,6 \%$ & $24,2 \%$ & $15,3 \%$ & $25,0 \%$ &, 847 \\
\hline 3. Maquetas & $29,2 \%$ & $23,4 \%$ & $15,6 \%$ & $31,8 \%$ &, 113 \\
\hline 4. Elaboración de un producto & $29,5 \%$ & $23,2 \%$ & $17,9 \%$ & $29,5 \%$ & ,248 \\
\hline 5. Cuestionarios & $30,7 \%$ & $22,8 \%$ & $15,2 \%$ & $31,4 \%$ &, 017 \\
\hline 6. Examen oral & $34,0 \%$ & $24,7 \%$ & $16,2 \%$ & $25,0 \%$ &, 380 \\
\hline $\begin{array}{l}\text { 7. Exposición oral de un tema inves- } \\
\text { tigado }\end{array}$ & $33,1 \%$ & $25,3 \%$ & $15,7 \%$ & $25,9 \%$ & ,225 \\
\hline $\begin{array}{l}\text { 8. Exámenes con preguntas de res- } \\
\text { puesta breve }\end{array}$ & $31,7 \%$ & $24,5 \%$ & $16,3 \%$ & $27,6 \%$ &, 333 \\
\hline $\begin{array}{l}\text { 9. Exámenes con preguntas de desa- } \\
\text { rrollo }\end{array}$ & $36,8 \%$ & $24,3 \%$ & $15,9 \%$ & $23,0 \%$ & ,415 \\
\hline $\begin{array}{l}\text { 10. Exámenes de preguntas "objeti- } \\
\text { vas" (opción múltiple, F/V, corres- } \\
\text { pondencia, etc.) }\end{array}$ & $35,8 \%$ & $20,9 \%$ & $14,2 \%$ & $29,1 \%$ & ,328 \\
\hline 11. Examen a libro abierto & $26,1 \%$ & $27,0 \%$ & $17,5 \%$ & $29,4 \%$ &, 008 \\
\hline 12. Autoevaluación & $25,9 \%$ & $25,4 \%$ & $16,6 \%$ & $32,2 \%$ &, 005 \\
\hline $\begin{array}{l}\text { 13. Coevaluación (evaluación entre } \\
\text { compañeros) }\end{array}$ & $30,7 \%$ & $22,0 \%$ & $12,7 \%$ & $34,7 \%$ &, 049 \\
\hline 14. Resolución de problemas & $33,3 \%$ & $25,2 \%$ & $14,5 \%$ & $27,0 \%$ &, 331 \\
\hline $\begin{array}{l}\text { 15. Diagramas, esquemas, mapas } \\
\text { conceptuales }\end{array}$ & $30,2 \%$ & $26,7 \%$ & $17,4 \%$ & $25,6 \%$ &, 322 \\
\hline 16. Portafolio & $19,4 \%$ & $29,0 \%$ & $19,4 \%$ & $32,3 \%$ &, 331 \\
\hline 17. Resumen o síntesis de contenidos & $35,0 \%$ & $22,8 \%$ & $15,7 \%$ & $26,4 \%$ & ,999 \\
\hline 18. Trabajos prácticos individuales & $32,1 \%$ & $23,5 \%$ & $17,1 \%$ & $27,3 \%$ &, 033 \\
\hline 19. Trabajos prácticos grupales & $34,3 \%$ & $23,2 \%$ & $16,2 \%$ & $26,3 \%$ & ,980 \\
\hline 20. Rúbrica de realimentación & $4,5 \%$ & $36,4 \%$ & $9,1 \%$ & $50,0 \%$ &, 004 \\
\hline $\begin{array}{l}\text { 21. Lista de cotejo/ observación de } \\
\text { desempeño }\end{array}$ & $25,5 \%$ & $20,0 \%$ & $12,7 \%$ & $41,8 \%$ &, 054 \\
\hline Otro & $31,1 \%$ & $24,3 \%$ & $17,6 \%$ & $27,0 \%$ & ,908 \\
\hline
\end{tabular}




\section{PRÁCTICAS Y CONCEPTO DE EVALUACIÓN PREVALENTES}

habían aprendido (representación tradicional) y en otro caso el colador se utilizó para explicar a la evaluación como una herramienta que orienta al docente acerca de sus prácticas de enseñanza eficaces o ineficaces para que los estudiantes aprendan, y el aprendizaje que queda pueda ser utilizado para seguir construyendo (representación constructivista). De las 522 encuestas, 57 casos fueron declarados inválidos para las preguntas abiertas, ya sea porque no respondieron o porque no interpretaron la consigna de manera que se pudiera catalogar las respuestas.

La Tabla 4 muestra las categorías que se construyeron a partir de las respuestas dadas, según su frecuencia de aparición. Las 10 categorías más frecuentes de representaciones mentales de la evaluación fueron las siguientes: medio para visibilizar y observar detalles; verificación de contenidos conceptuales; detección de errores, nivelación y corrección; mejora, cambio y oportunidad; seguimiento de un proceso; medición; conocimiento profundo del estudiante; construcción; autoevaluación; aprendizaje.

\section{Tabla 4}

Frecuencia de aparición de categorias de las representaciones mentales de la evaluación $(N=465)$

\begin{tabular}{lcc}
\hline \multicolumn{1}{c}{ La evaluación es vista como... } & $n$ & $\%$ \\
\hline Medio para visibilizar y observar detalles & 65 & 14,0 \\
Verificación de contenidos conceptuales & 50 & 10,8 \\
Detección de errores, nivelación y corrección & 35 & 7,5 \\
Mejora, cambio y oportunidad & 34 & 7,3 \\
Seguimiento de un proceso & 30 & 6,5 \\
Medición & 27 & 5,8 \\
Conocimiento profundo del estudiante & 27 & 5,8 \\
Construcción & 25 & 5,4 \\
Autoevaluación & 22 & 4,7 \\
Aprendizaje & 20 & $4,3 \%$ \\
Descubrimiento de las diferencias individuales & 19 & $4,2 \%$ \\
Como integrador de las capacidades & 18 & $3,9 \%$ \\
Comprensión & 16 & $3,4 \%$ \\
Guía hacia las metas & 14 & $3 \%$ \\
Diagnóstico & 14 & $3 \%$ \\
Medio de expresión & 10 & $2,2 \%$ \\
Crecimiento & 8 & $1,7 \%$ \\
Proceso creativo & 6 & $1,3 \%$ \\
Resaltador de logros & 6 & $1,3 \%$ \\
Rutina de la educación & 5 & $1,1 \%$ \\
Descubrimiento de fortalezas y debilidades & 3 & $0,6 \%$ \\
Depurador o decantador de los que no llegan & 3 & $0,6 \%$ \\
El final de un proceso & 3 & $0,6 \%$ \\
Revisión o repaso & 3 & $0,6 \%$ \\
Complejidad & 2 & $0,4 \%$ \\
\hline
\end{tabular}


Las categorías fueron recategorizadas, a su vez, en dos grandes grupos o paradigmas: las que describen un concepto conductista/tradicional, de medición y con un enfoque predominantemente cuantitativo, y las que describen un concepto alineado con la evaluación constructivista, auténtica, con un enfoque más cualitativo. En la Tabla 5 se pueden apreciar las categorías reagrupadas. Tal como se observa, los porcentajes indican que el concepto que aparece en las representaciones mentales de los docentes está dividido entre ambos enfoques, siendo el enfoque auténtico algo más frecuente que el tradicional.

A fin de entender las conceptualizaciones que predominan, se presentan algunos ejemplos de objetos y explica- ciones de ambos enfoques que fueron declarados por los docentes.

\section{Enfoque tradicional de la evaluación}

Para este enfoque, se seleccionaron dos categorías a modo de ejemplos: medición y verificación de contenidos conceptuales.

La categoría "medición" se construyó a partir de las respuestas de los docentes que utilizaron objetos tales como la regla, la balanza, la cinta de medir, el nivel de albañil, entre otros, asociados a explicaciones tales como "porque me permite medir los conocimientos de los alumnos y compararlos" y "porque va midiendo los logros alcanzados por cada alumno, y al ir sumando cada logro se va haciendo más largo el centímetro".

Tabla 5

Categorías reagrupadas en dos enfoques o paradigmas de la evaluación

\begin{tabular}{|c|c|c|c|c|c|}
\hline \multicolumn{3}{|c|}{ Enfoque tradicional/conductista } & \multicolumn{3}{|c|}{ Enfoque constructivista } \\
\hline Categoría & $n$ & $\%$ & Categoría & $n$ & $\%$ \\
\hline \multirow{2}{*}{$\begin{array}{l}\text { Medio para visibilizar y obser- } \\
\text { var detalles }\end{array}$} & \multirow{2}{*}{65} & \multirow{2}{*}{14} & Mejora, cambio y oportunidad & 34 & 7,3 \\
\hline & & & Seguimiento de un proceso & 30 & 6,5 \\
\hline $\begin{array}{l}\text { Verificación de contenidos } \\
\text { conceptuales }\end{array}$ & 50 & 10,8 & $\begin{array}{l}\text { Conocimiento profundo del } \\
\text { estudiante }\end{array}$ & 27 & 5,8 \\
\hline \multirow{2}{*}{$\begin{array}{l}\text { Detección de errores, nivelación } \\
\text { y corrección }\end{array}$} & \multirow[t]{2}{*}{35} & \multirow[t]{2}{*}{7,5} & Construcción & 25 & 5,4 \\
\hline & & & Autoevaluación & 22 & 4,7 \\
\hline Medición & 27 & 5,8 & Aprendizaje & 20 & 4,3 \\
\hline Guía hacia las metas & 14 & 3,0 & $\begin{array}{l}\text { Descubrimiento de las diferen- } \\
\text { cias individuales }\end{array}$ & 19 & 4,2 \\
\hline Rutina de la educación & 5 & 1,1 & Como integrador de las capa- & & \\
\hline \multirow{2}{*}{$\begin{array}{l}\text { Descubrimiento de fortalezas y } \\
\text { debilidades }\end{array}$} & \multirow{2}{*}{3} & \multirow{2}{*}{0,6} & cidades & 18 & 3,9 \\
\hline & & & Comprensión & 16 & 3,4 \\
\hline \multirow{2}{*}{$\begin{array}{l}\text { Depurador o decantador de los } \\
\text { que no llegan }\end{array}$} & \multirow[t]{2}{*}{3} & \multirow{2}{*}{0,6} & Diagnóstico & 14 & 3,0 \\
\hline & & & Medio de expresión & 10 & 2,2 \\
\hline El final de un proceso & 3 & \multirow{2}{*}{$\begin{array}{l}0,6 \\
0,6\end{array}$} & Crecimiento & 8 & 1,7 \\
\hline \multirow[t]{2}{*}{ Revisión o repaso } & \multirow[t]{2}{*}{3} & & Proceso creativo & 6 & 1,3 \\
\hline & & & Complejidad & 2 & 0,4 \\
\hline Totales & 214 & 46,0 & & 251 & 54,0 \\
\hline
\end{tabular}


La categoría "verificación de contenidos conceptuales" se formó en torno de objetos, como radiografía, lupa, llave, entre otros, vinculados a explicaciones tales como "porque ayuda a verificar lo que aprendieron de cada contenido", "porque con la radiografía puedo ver más de lo que se ve a simple vista, más en profundidad el alcance del conocimiento de los contenidos" y "porque a partir de los contenidos evaluados me va a permitir dar otros nuevos".

El análisis de las respuestas categorizadas bajo el enfoque tradicional permite observar la evaluación centrada en el contenido y en la figura del docente. En general, estas respuestas no mencionan el aprendizaje, el estudiante y la mejora a partir de la evaluación.

\section{Enfoque de evaluación constructivista}

Para este enfoque, se seleccionaron dos categorías a modo de ejemplo: aprendizaje y seguimiento de un proceso.

La categoría "aprendizaje" se conformó a partir de las respuestas de los docentes que utilizaron objetos tales como la llave, el pincel y otros, asociados a explicaciones, como por ejemplo,

la llave representa la evaluación porque no es la etapa final del aprendizaje, sino que es la que nos permite abrir y avanzar hacia nuevos aprendizajes, más complejos. También permite, cuando no fue logrado el aprendizaje, abrirnos hacia otras estrategias y modalidades. Nos lleva a conocer el proceso de desarrollo de las habilidades de cada alumno.

O como la siguiente: "La llave representa mi concepto de evaluación porque permite al alumno y me permite a mí seguir abriendo puertas para el aprendizaje y la superación personal de cada uno".

Para la conformación de la categoría "seguimiento de un proceso", los objetos elegidos y asociados a sus respectivas explicaciones fueron muy variados: lupa, llave, nivel de albañil, pincel, esponja, brújula, faro, entre otros. Algunas explicaciones: "Una llave sirve para abrir, básicamente. La evaluación es una herramienta que abrirá múltiples posibilidades, porque permite ver el proceso de los aprendizajes en sus distintas etapas, buscando resultados válidos, fiables y eficientes".

Elijo el nivel de albañil porque considero que la evaluación se da de manera procesual, y que el nivel no se utiliza solamente al final de la construcción, sino que se utiliza para retroalimentar el proceso de evaluación durante toda la construcción, permitiendo identificar aspectos de mejora, como también fortalezas (aquello que se logró y se hizo bien). En estas categorías, como en las otras que se construyeron a partir de las respuestas de los docentes, se puede percibir un concepto de evaluación más cercano a las instancias de aprendizaje, realimentación y uso del error como una fuente de aprendizaje, características de una evaluación formativa, constructiva y auténtica.

\section{Las categorías menos frecuentes}

En su conjunto, otras categorías encontradas, también aportan comprensión a la manera en que los docentes entienden la evaluación. Para algunos, la evaluación es una forma de descubrir las diferencias individuales de sus estudiantes y de respetarlas. También les permite integrar todas las capacidades de sus alumnos, les ayuda a comprender mejor lo que sucede con el aprendizaje, 
es una guía hacia las metas, es un buen diagnóstico, es un medio de expresión $\mathrm{y}$ es una forma de crecimiento. Hay quienes la consideran un proceso creativo y una forma de resaltar logros. Por otro lado, también hay docentes que la entienden como una rutina de la educación, una forma de descubrir fortalezas y debilidades y un depurador o decantador de los que no llegan. Otros se representan como el final de un proceso, una forma de revisión o repaso, o como complejidad.

\section{Discusión}

En la revisión de los datos obtenidos para este estudio, se consideraron para el presente informe solamente los relacionados a las prácticas más frecuentes de evaluación y a la conceptualización de la evaluación.

El objetivo de este trabajo ha sido relevar las prácticas de evaluación más frecuentes de docentes de nivel primario y secundario y los conceptos de evaluación predominantes expresados en sus representaciones sociales del concepto.

En cuanto a las prácticas más frecuentes, se encontró que los docentes del primer ciclo de educación primaria usaban más los exámenes escritos con ejercicios similares a los dados en clases, los trabajos prácticos individuales, los exámenes orales, la resolución de problemas y las exposiciones orales de un tema investigado. Un análisis detallado y cualitativo de estas prácticas de evaluación recién permitiría dirimir si están alineadas con un enfoque conductista o constructivista. Sin embargo, como estrategias en su conjunto, no parecen estar inclinadas hacia una evaluación de competencias, sino más bien centradas en el contenido.

En el segundo ciclo de educación primaria no hay demasiadas diferencias. Las prácticas más frecuentes de evaluación fueron los trabajos prácticos individuales, los exámenes escritos con ejercicios para resolver, similares a los dados en clases, los exámenes orales, las exposiciones orales de un tema investigado y la resolución de problemas.

En el nivel secundario, los docentes utilizan más frecuentemente los trabajos prácticos individuales, los trabajos prácticos grupales, los exámenes escritos con ejercicios para resolver, similares a los dados en clase, las exposiciones orales de un tema investigado y los exámenes orales.

La práctica menos utilizada, en general, fue el portafolio. Esta es una herramienta de proceso, que sigue la trayectoria en la adquisición de competencias.

Un análisis de $\chi^{2}$ mostró que en el primer ciclo de la educación primaria se utilizan, más que en los otros niveles, los dictados y maquetas. Este resultado no sorprende, pues una manera de ejercitar y evaluar los logros de la alfabetización es el dictado, una herramienta efectiva para este objetivo.

Las maquetas, por su parte, son estrategias que permiten observar la integración de habilidades manuales, espaciales y conceptuales, que se desarrollan desde el nivel inicial. Ambas prácticas pueden brindar elementos para tener una evaluación de competencias.

Pero en el primer ciclo de la educación primaria hay menos variedad de prácticas de evaluación declaradas. Esto también muestra una mayor tendencia a la evaluación de conceptos que de competencias.

En el nivel secundario se utilizan, más que en los otros niveles, prácticas tales como la elaboración de un producto, los cuestionarios, los exámenes con 
preguntas de desarrollo, los exámenes con preguntas objetivas, los exámenes a libro abierto, la coevaluación, la resolución de problemas, los diagramas/esquemas/mapas conceptuales, el resumen o síntesis de contenidos, los trabajos prácticos individuales, los trabajos prácticos grupales, la rúbrica de realimentación. La variedad de prácticas tal vez esté relacionada con la diversidad de asignaturas y docentes a cargo, que seleccionan las más adecuadas para la evaluación de sus áreas específicas. De la misma manera que en el análisis anterior, se encuentra que dichas prácticas son apropiadas para evaluar competencias si se usan en forma efectiva.

Esta variedad de prácticas enriquece la evaluación formativa y proporciona una visión más integral y completa del trayecto recorrido por los estudiantes. En el pensamiento de Santos Guerra (2007), la diversidad de instrumentos para evaluar a un sujeto le añade rigor al proceso.

En cuanto a la relación entre los años de experiencia de los docentes y las prácticas más usuales, se encontró que quienes tienen menos de cinco años de experiencia tienden a utilizar menos el examen a libro abierto, la autoevaluación y la coevaluación que los de los demás rangos de experiencia. Este hallazgo podría estar relacionado con cierta inseguridad en el manejo de prácticas que requieren habilidad para darle cierto rigor y validez. Tal vez posteriormente, al afirmar sus competencias, encuentren la manera de incorporar estas prácticas efectivas para tener un cuadro más completo de lo que se desea evaluar. Por otro lado, quienes tienen más de 15 años de experiencia tienden a utilizar más los cuestionarios, los trabajos prácticos individuales y las rúbricas que los de los otros rangos de años de experiencia. Los cuestionarios y los trabajos prácticos individuales son prácticas más tradicionales, con lo cual se podría pensar que estos docentes siguen aplicando aquellas estrategias con las que fueron evaluados. El uso de rúbricas es una práctica más reciente en la educación y requiere de ciertas habilidades de elaboración, que evidentemente han incorporado estos docentes con mayor experiencia. Una de las funciones de la evaluación, según Elola et al. (2010), es el desarrollo de capacidades y competencias valiosas, tanto individuales, como colectivas e institucionales. En este contexto, es positivo notar que los docentes de mayor experiencia hayan incorporado una práctica nueva y compleja a sus habilidades de evaluación.

El análisis cualitativo mostró, en forma general, las representaciones mentales de los docentes acerca del concepto de evaluación. Dado que las creencias impactan directamente en el hacer, es importante reconocer cuáles son las representaciones predominantes (Moreno Acero et al., 2016).

Se pudieron construir 25 categorías a partir de las explicaciones que los docentes dieron de los objetos que eligieron para representar el concepto. De esas 25 categorías iniciales, se destacan 10 que fueron las más frecuentes. Así se representó a la evaluación como medio para visibilizar y observar detalles; verificación de contenidos conceptuales; detección de errores, nivelación y corrección; mejora, cambio y oportunidad; seguimiento de un proceso; medición; conocimiento profundo del estudiante; construcción; autoevaluación y aprendizaje.

Estas 10 categorías pueden recategorizarse en prácticas conductistas y constructivistas. Dentro de la categoría 
conductista hubo mayor cantidad de representaciones $(n=174)$, con poca diferencia para la categoría constructivista $(n=158)$. Sin embargo, en el recuento total de las 10 categorías más frecuentes, sumadas a las 15 restantes, el $54 \%$ de la población manifestó representaciones tendientes a un enfoque constructivista, mientras que el $46 \%$ restante adhirió a explicaciones que muestran un enfoque conductista y tradicional.

Las categorías enfocadas en una evaluación auténtica y constructivista se centraron mayormente en las funciones políticas, de conocimiento, de mejoramiento y de desarrollo de capacidades, según la conceptualización de Elola et al. (2010), mientras que las categorías enfocadas en una evaluación conductista y tradicional atendieron mayormente a las funciones simbólica y contractual, según los mismos autores.

Esta división en partes casi equilibras revela la tensión que existe en las representaciones mentales acerca de la evaluación. Coexisten modelos tradicionales en escuelas que han iniciado un cambio y que intentan ofrecer propuestas de evaluación coherentes con las innovaciones curriculares y didácticas.

Puede explicarse también con docentes que conocen las nuevas prácticas de evaluación para considerar el logro de competencias, pero por razones de comodidad y seguridad recurren a las viejas prácticas con las que fueron evaluados cuando fueron estudiantes o las que han utilizado por muchos años. Por otra parte, las prácticas tradicionales son más fáciles de sostener ante los padres, que pueden no estar preparados para entender los sistemas de evaluación enfocados en competencias.

La tensión entre conceptos queda ejemplificada con algunos casos en los que los objetos que fueron escogidos para representar a la evaluación no son los tradicionales (no eligieron el centímetro, nivel de albañil, metro, etc.) pero en cuya explicación se transluce un concepto tradicional de la evaluación. Un ejemplo de este hecho se observa en esta respuesta:

Para mí el concepto de evaluación es como un espejo, el cual refleja cómo están los alumnos de acuerdo de los contenidos desarrollados. Puedo ver si hay un problema, reflexionar sobre ello y buscar solución, ya sea la estrategia usada para presentar los contenidos o el método usado para evaluar o si la consigna no fue clara.

El centro del problema aparece en el contenido. Después se añade la reflexión, para volver a centrarse en los contenidos y por último surgen las estrategias de evaluación. Quien da esta explicación evidentemente tiene el concepto en tensión y todavía no opta por una representación constructivista de la evaluación.

Por otra parte, hubo docentes que respondieron con mucha convicción dando una respuesta que muestra un concepto de evaluación auténtica, con un enfoque constructivo. Un ejemplo de ello es esta respuesta:

Elijo las agujas de tejer, porque entiendo a la evaluación como un proceso de mejora continua, de la misma manera que cuando tejemos hacemos y deshacemos puntos hasta lograr una pieza de tejido perfecta, a través de utilizar tiempo, esfuerzo, dedicación y el desarrollo de nuestra destrezas, conocimiento y habilidades, también el proceso de evaluación muestra los avances y retrocesos de los alumnos y de aquellos contenidos que debemos ajus- 


\section{PRÁCTICAS Y CONCEPTO DE EVALUACIÓN PREVALENTES}

tar o rever para lograr un resultado óptimo al finalizar el ciclo escolar. Y por otro lado también autoevaluamos nuestra función como artífices de ese entramado que vamos tejiendo a lo largo del año y vemos, por supuesto aquellos ajustes o mejoras que debemos hacer en nuestra quehacer cotidiano.

En esta última representación encontramos que la evaluación no es un apéndice de la enseñanza, sino una parte integral del proceso, como prescribe Álvarez Méndez (2000) y tiene la función de mejoramiento (Elola, et al. 2010).

Las restantes categorías encontradas, en su conjunto, también aportan comprensión a la manera en que los docentes entienden a la evaluación, que en muchos casos describen una evaluación auténtica y constructivista.

Las prácticas más utilizadas deberían ser una demostración de estas conceptualizaciones. Sin embargo, como se ha mostrado en los análisis de datos, las prácticas más frecuentes tienden a ser más bien tradicionales. Por esto se puede afirmar que las representaciones mentales de la evaluación que tienen los docentes tienen un enfoque más constructivista que sus prácticas reales.

El propósito de este estudio ha sido aportar comprensión acerca de cómo se entiende y practica la evaluación en los niveles primario y secundario. Más estudios como este ayudarán a ampliar esa comprensión y pueden ofrecer una base para escoger líneas de intervención y mejora de la evaluación educacional.

\section{Referencias}

Ahumada, A. P. (2005). La evaluación auténtica: un sistema para la obtención de evidencias y vivencias de los aprendizajes. Perspectiva Educacional, Instituto de Educación PUCV, 45(1), 11-24.
Álvarez Méndez, J. M. (2000). Didáctica, currículo y evaluación: ensayos sobre cuestiones didácticas. Buenos Aires: Miño y Dávila.

Anijovich, R. y Cappelletti, G. (2017). La evaluación como oportunidad. Buenos Aires: Paidós.

Anijovich, R. y González, C. (2011). Evaluar para aprender: conceptos e instrumentos. Buenos Aires: Aique.

Arbeláez Gómez, M. (2001). Las representaciones mentales. Revista de Ciencias Humanas, 8(29), 87-94. Recuperado de https://www.academia .edu/5121611/_Las_representaciones_mentales_

Cabezas Gómez, D., González Barberá, C. y Carpintero Molina, E. (2009). Evaluar la evaluación: cuestionario sobre prácticas de evaluación. EduPsykhé, 8(1), 51-61.

Elola, N., Zanelli, N., Oliva, A. y Toranzos, L. (2010). La evaluación educativa: fundamentos teóricos y orientaciones prácticas. Buenos Aires: Aique.

Moreno Acero I. D., Lozano Ramírez, A. D., Pineda Ramos, C. A. y Ortíz Quiróz, J. F. (2016). Representaciones sociales sobre el rol de la familia en la escuela que construyen los estudiantes y las familias. Horizontes Pedagógicos, 18(2), 52-65.

Mellado Hernández, M. E. y Chaucono Catrinao, J. C. (2015). Creencias pedagógicas del profesorado de una escuela rural en el contexto mapuche. Actualidades Investigativas en Educación, 15(3). https://doi.org/10.15517/aie .v15i3.20924.

Perkins, D. (2010). El aprendizaje pleno: principios de la enseñanza para transformar la educación. Buenos Aires: Paidós.

Prieto, M. y Contreras, G. (2008). Las concepciones que orientan las prácticas evaluativas de los profesores: un problema a develar. Estudios Pedagógicos, 34(2), 245-262. https://doi .org/10.4067/S0718-07052008000200015

Rafaghelli, M. (2009). ¡Malditos referentes! Sobre la necesidad de reflexionar en torno a los referentes en las prácticas evaluadoras. Itinerarios Educativos, 1(3), 86-104. https://doi .org/10.14409/ie.v1i3 .3917

Santos Guerra, M. A. (2007). La evaluación como aprendizaje: una flecha en la diana. Buenos Aires: Narcea.

Santos Guerra, M. A. (2017). Evaluar con el corazón: de los ríos de las teorías al mar de la práctica. Buenos Aires: Homo Sapiens.

Señoriño, O. A., Vilanova, S. L., García, M. B., Natal, M. y Lynch, M. I. (2012). Concepciones sobre evaluación en profesores en formación: un estudio comparativo entre alumnos de profesorado de las facultades de la Universidad Nacional de Mar del Plata, 


\section{KRUMM, CAIRUS Y QUINDE}

Argentina. Revista de Evaluación Educativa, 1(2). Recuperado de https://www.academia .edu/33304586

Vallejo Ruiz, M. y Molina Saorín, J. (2014). La evaluación auténtica de los procesos educativos. Revista Iberoamericana de Educación, 64, 11-25. https://doi.org/10.35362/rie640403

Zabalza, M.A. (2014, abril). La evaluación en la universidad y en el nivel superior: un proce- so cargado de tensiones y de oportunidades. Ponencia presentada en el VIII Congreso Iberoamericano de Docencia Universitaria, Rosario, Argentina.

Recibido: 20 de noviembre de 2018

Revisado: 15 de febrero de 2019

Aceptado: 29 de marzo de 2020 